\title{
Commentary
}

\section{Research Progress of Metformin in the Treatment of Non-alcoholic Fatty Liver Disease}

\author{
Chengsheng Liu ${ }^{1}$, Jiehua Xie ${ }^{2, \text { * }}$ \\ ${ }^{1}$ Health Center of Huashan Town, Guangzhou, China \\ ${ }^{2}$ District Center for Disease Control and Prevention, Guangzhou, China \\ Email address: \\ 340419781@qq.com (Chengsheng Liu),Joky71797@126.com (Jiehua Xie) \\ ${ }^{*}$ Corresponding author
}

\section{To cite this article:}

Chengsheng Liu, Jiehua Xie. Research Progress of Metformin in the Treatment of Non-alcoholic Fatty Liver Disease. Advances in Bioscience and Bioengineering. Vol. 8, No. 4, 2020, pp. 78-81. doi: 10.11648/j.abb.20200804.13

Received: December 6, 2020; Accepted: December 16, 2020; Published: December 22, 2020

\begin{abstract}
Backgroud: Nonalcoholic fatty liver disease has become the second largest source of chronic liver disease in the urban population. Metformin, a traditional drug, has good prospects for treating the disease in primary health care facilities. Objective: To summarize and grasp the status of metformin in adults, children with diabetes and non-diabetic NAFLD patients. Methods: We reviewed 31 relevant research literatures in PubMed database, and sorted out some preclinical and clinical research results. Results: Metformin was found to have a good effect in improving liver function indicators in both adult diabetic and non-diabetic NAFLD patients, and could benefit liver histology in NAFLD patients with adult diabetes. Metformin treatment did not show a significant advantage over lifestyle interventions in children with NAFLD. Conclusion: Metformin can improve the liver function and histological structure of adult NAFLD patients to a certain extent, and children patients should still be treated mainly with improved lifestyle.
\end{abstract}

Keywords: Non-alcoholic Fatty Liver Disease, Metformin, Diabetes

\section{Introduction}

The nonalcoholic fatty liver disease (Non-alcoholic fatty liver disease, NAFLD) showed a trend of growing worldwide, has become a common after HBV chronic liver disease. The prevalence is between $20 \%$ and $46 \%$ in developed countries in Europe and the Americas [1]. The prevalence of nonalcoholic fatty liver disease in developing countries represented by China in Asia is between 5\% and 40\% [2]. The prevalence is highest in people aged 40 to 49 years [3]. This is the main cause of chronic liver disease in obese children [4].

The routine management of NAFLD will be left to a wide range of primary health-care institutions. NAFLD can be classified into non-alcoholic fatty liver (NAFLD) or non-alcoholic steatohepatitis (NASH) at two different clinical stages according to different pathological changes [5]. Some patients are concurrent with type 2 diabetes. Liver failure, cirrhosis, and hepatocellular carcinoma are the terminal adverse outcomes of NASH [6]. Standardized drug intervention in early primary medical institutions plays a key role in stopping the occurrence of adverse outcomes. However, access to drugs and limited medical economy are the difficulties for primary medical institutions to carry out diagnosis and treatment. Therefore, the study on the pharmacological mechanism of traditional drugs in improving the prognosis of NAFLD is the focus of attention of doctors at the grassroots level.

The pathogenesis of NAFLD is not fully understood, and the pathophysiological "double strike" hypothesis has been gradually proposed: the "first strike" is the synergistic effect of obesity and diet, and the "second strike" is the mechanism of inflammation and cell damage. Insulin resistance is known to play a key role in the development of disease, and the "second strike" is an important reason for aggravating insulin resistance [7, 8]. Insulin resistance activates adipogenic transcription factor steroregulatory factor binding protein-1 (srebp-1) through the AMPK pathway to promote liver 
adipogenesis, while peripheral adipoolysis leads to increased flow of free fatty acids into the liver, leading to pathological changes in liver cell structure $[9,10]$. In addition to adjusting diet and lifestyle, metformin is an insulin sensitizer that has been shown to improve insulin resistance and is a first-line oral therapy recommended by global guidelines for the treatment of type 2 diabetes mellitus (T2DM) [11, 12], which is readily available at all levels of medical centers. Multiple studies have comfirmed the beneficial effects of metformin on the biochemical or histological characteristics of non-alcoholic fatty liver disease [13-16]. Metformin has also been shown to regulate the synthesis of tumor necrosis factor and interleukin- 6 to increase -oxidation of free fatty acids and reduce de novo synthesis to prevent fat accumulation in the liver [9, 10, 17].

As a result, metformin has been widely recommended in several guidelines for the treatment of adult NAFLD with type 2 diabetes. However, will the drug benefit from its use in non-diabetic patients and at different ages, especially in children with NAFLD? Can metformin improve NASH organizational change? How is the dose of metformin controlled in the course of different diseases? It is still a difficult problem that needs urgent attention in clinical practice. Therefore, the purpose of this review is to summarize adult and pediatric studies, and to classify clinical studies of metformin in diabetic and non-diabetic NAFLD patients, including the results of liver biopsy after treatment, so as to provide more detailed basic data for the promotion and application of metformin in primary clinical NAFLD treatment.

\section{Preclinical Study}

Early evidence of metformin's benefit in the treatment of NAFLD came from preclinical studies in insulin-resistant mice with nonalcoholic fatty liver disease $[13,18]$. Lin and $H$. $\mathrm{Z}$ et al found for the first time in mouse model that metformin improved fatty liver disease, reversed hepatocellular pathological changes, and normalized Alt levels, indicating that metformin could not only prevent and reverse the development of steatosis, but also prevent and reverse liver inflammation [13, 18]. Although metformin has shown significant therapeutic effects in mice with NAFLD, more clinical studies are needed to further validate it.

\section{Clinical Study}

\subsection{Adult Diabetes}

Because T2DM and nonalcoholic fatty liver share similar pathogenesis, T2DM appears as an important complication in many cases of nonalcoholic fatty liver disease. Metformin has sufficient indications for the treatment of T2DM, but whether NASH patients combined with T2DM can further obtain liver benefits and how the dose-response relationship is an issue to be discussed in clinical studies.

A small dose of metformin $(1 \mathrm{~g} / \mathrm{d})$ for half a year compared with diet showed that both of them could improve liver metabolic parameters, confirming the priority of improving diet structure and living habits. However, small dose of metformin could improve liver function more significantly. In addition, liver steatosis was significantly improved in both groups [19]. Similar results were shown in another clinical trial with a low dose of metformin (850-1000 mg/d) [20]. These two studies suggest that a small dose of metformin can benefit liver function. However, a study that increased the dose of metformin to $2000 \mathrm{mg}$ /day showed that only $30 \%$ of patients experienced histological reactions and improvements in alanine aminotransferase levels, which were associated with weight loss [21]. This suggests that increasing the dose of metformin does not necessarily lead to greater benefits. In addition, a 19-person randomized, placebo-controlled diet, exercise, and metformin diet and exercise trial showed no improvement in histology or liver enzyme levels in either group [22].

Omer et al [22] of 64 patients receiving metformin, combination treatment with rosiglitazone single-agent or both studies, the results show that metformin group is the only beneficial effects of body mass index falls significantly without changing the liver fat content and ALT levels increase hepatic insulin sensitivity, and the results of another study [23] Nair et al [24] conducted post-treatment biopsies on 10 participants, after 3 months of treatment, insulin resistance improved, corresponding to a decrease in the levels of ALT and AST. However, during the study, insulin sensitivity was stable and transaminase increased gradually again. The histological changes were very slight. Loomba et al [21] studied the efficacy of metformin in NASH patients for 48 weeks, and the results showed that patients could obtain a corresponding tissue response with weight loss, and reduce hepatocyte injury and parential inflammation accordingly. Bugianesi et al [25] studied 110 patients with nonalcoholic fatty liver disease who were randomly given metformin or a control group (vitamin E or diet) for 12 months. ALT levels were significantly reduced in metformin patients, and follow-up biopsies of 17 patients showed improvements in steatosis, necroinflammation, fibrosis, and NASH index. Interestingly, a recent large case-control study showed that the use of metformin was dose-dependent with the reduction of HCC risk in diabetic patients [26]. The authors found a 7\% reduction in $\mathrm{HCC}$ risk for each additional year of use. These results suggest that metformin in patients with nonalcoholic fatty liver disease may have a beneficial effect of ideal, small doses of metformin (800-1000 mg), liver function can obtain benefit, most studies support metformin treatment for NASH reversible hepatocyte steatosis, overall improve the histological structure, reduce inflammation and reduce NASH to the risk of $\mathrm{HCC}$, liver failure and liver cirrhosis transformation.

\subsection{Non-diabetic}

Although current guidelines and the above studies support the recommendation of metformin for the treatment of nonalcoholic fatty liver disease with T2DM. However, 
whether patients with nonalcoholic fatty liver disease without T2DM can benefit from it is a key issue we need to discuss. Uygun et al. [24] randomly selected 36 of T2DM patients with $\mathrm{NASH}$, respectively treated with diet or diet plus metformin alone for six months, the results showed that the BMI of join the metformin group, aspartate aminotransferase (AST), alanine aminotransferase (ALT) and insulin resistance index were improved significantly, necrotizing inflammation activity declined slightly, but the results did not reach statistical significance. Idilman et al. also demonstrated in a study of 74 NASH patients that diet plus metformin resulted in significant weight loss, reduced Alt, and insulin resistance, but no histological improvement. These studies suggest that metformin treatment of nonalcoholic fatty liver disease without T2DM can also reduce liver inflammatory markers and insulin resistance, but does not show significant histologic benefit in this group of patients. More evidence is needed to support the effect of metformin on liver histology in non-diabetic patients [8].

\subsection{In Children}

The paradoxes of the above studies point to the low dose of metformin used and the lack of significant improvement in insulin sensitivity may be the key reasons for the poor histologic changes. Although metformin has been shown to be effective in adult studies, it has shown different results in children. Two pediatric trials have been conducted [29, 30]. Nobili et al. evaluated the effect of diet plus metformin on nonalcoholic fatty liver disease in children aged 9-18 years for 2 years and found that metformin did not appear to be more effective than lifestyle interventions in improving transaminase levels, steatosis, and liver histology in children with NAFLD. Second study is by far the largest pediatric research, evaluated the 173 ages 8 to 17 patients with nonalcoholic fatty liver disease, they accepted the 96 weeks of vitamin E, metformin, or placebo, no a set of ALT levels continued to decline, the study has not been reported in the use of metformin in children with diabetes, any significant adverse events. These studies suggest that the focus of treatment for childhood NAFLD is still to improve lifestyle and adjust diet.

\section{Conclusion}

The proportion of patients with non-alcoholic fatty liver disease shows a trend of continuous growth with economic development, especially in economically developed urban and rural areas, and is also the most common cause of chronic liver disease in children and adolescents. The diagnosis and treatment of NAFLD will be left to the majority of primary medical institutions. Liver failure, cirrhosis, and hepatocellular carcinoma are the terminal adverse outcomes of NAFLD [6]. Standardized and effective drug intervention therapy in early primary medical institutions plays a key role in ending the occurrence of adverse outcomes. Understanding the pharmacological mechanism of traditional drugs in improving the prognosis of
NAFLD is the focus of grassroots doctors.

This review classifies and sorts out the relevant clinical research data in the early stage, and compares the research results of patients with and without diabetes, adults and children. It was concluded that metformin in the treatment of nonalcoholic fatty liver disease, higher dose may be more likely to cause weight loss, reduce insulin resistance, and thus improve liver histology [27-28]. Two studies using higher doses of metformin did find significant weight loss [20-21]. The dose of metformin was 2.5-3 g/day, with an average weight loss of $4 \mathrm{~kg}$ in 6 months, while the dose of metformin was $2 \mathrm{~g} /$ day, with an average weight loss of $6 \mathrm{~kg}$ in 1 year [20-21]. Only mild gi side effects were reported in all the studies, and only two had a patient drop out because the side effects were intolerable [20]. No cases of lactic acidosis were detected, and treatment for adults and children was safe, but treatment for children was not superior to lifestyle interventions.

Although metformin is effective in treating adult NAFLD patients with diabetes, it is still insufficient to undermine the role of lifestyle intervention in treatment, especially for children.

Although there have been many studies on metformin treatment of nonalcoholic fatty liver disease, few have been able to break down the differences between patients of different age groups and specific groups, especially children and pregnant women. It is believed that more and more detailed stratified research data will guide the clinical practice in the future.

\section{Acknowledgements}

Thank you to my roommates in the medical college as follow: Yangshen He, Luxin Chen, Hanjun Chen, Zhenqiang Chen, Yongliang Chen. In addition, I am especially grateful to Jiaxiong Tan for the help in the design and writing of this article.

\section{References}

[1] Ross AB, Godin JP, Minehira K, et al. Increasing whole grain intake as part of prevention and treatment of nonalcoholic Fatty liver disease [J]. International journal of endocrinology 2013, 2013: 585876.

[2] Amarapurkar DN, Hashimoto E, Lesmana LA, et al. How common is non-alcoholic fatty liver disease in the Asia-Pacific region and are there local differences? [J]. Journal of gastroenterology and hepatology 2007, 22 (6): 788-793.

[3] Paschos P, Paletas K. Non alcoholic fatty liver disease and metabolic syndrome [J]. Hippokratia 2009, 13 (1): 9-19.

[4] Loomba R, Sirlin CB, Schwimmer JB, et al. Advances in pediatric nonalcoholic fatty liver disease [J]. Hepatology (Baltimore, Md) 2009, 50 (4): 1282-1293.

[5] Kleiner DE, Brunt EM, Van Natta M, et al. Design and validation of a histological scoring system for nonalcoholic fatty liver disease [J]. Hepatology (Baltimore, Md) 2005, 41 (6): 1313-1321. 
[6] Starley BQ, Calcagno CJ, Harrison SA. Nonalcoholic fatty liver disease and hepatocellular carcinoma: a weighty connection [J]. Hepatology (Baltimore, Md) 2010, 51 (5): $1820-1832$.

[7] Day CP, James OF. Steatohepatitis: a tale of two "hits"? [J]. Gastroenterology 1998, 114 (4): 842-845.

[8] Chalasani N, Younossi Z, Lavine JE, et al. The diagnosis and management of non-alcoholic fatty liver disease: practice Guideline by the American Association for the Study of Liver Diseases, American College of Gastroenterology, and the American Gastroenterological Association [J]. Hepatology (Baltimore, Md) 2012, 55 (6): 2005-2023.

[9] Li Y, Xu S, Mihaylova MM, et al. AMPK phosphorylates and inhibits SREBP activity to attenuate hepatic steatosis and atherosclerosis in diet-induced insulin-resistant mice [J]. Cell metabolism 2011, 13 (4): 376-388.

[10] Mazza A, Fruci B, Garinis GA, et al. The role of metformin in the management of NAFLD [J]. Experimental diabetes research 2012, 2012: 716404

[11] Nathan DM, Buse JB, Davidson MB, et al. Medical management of hyperglycemia in type 2 diabetes: a consensus algorithm for the initiation and adjustment of therapy: a consensus statement of the American Diabetes Association and the European Association for the Study of Diabetes [J]. Diabetes care 2009, 32 (1): 193-203.

[12] Adler AI, Shaw EJ, Stokes T, et al. Newer agents for blood glucose control in type 2 diabetes: summary of NICE guidance [J]. BMJ (Clinical research ed) 2009, 338: b1668.

[13] Mantovani A, Byrne CD, Scorletti E, Mantzoros CS, Targher G. Efficacy and safety of anti-hyperglycaemic drugs in patients with non-alcoholic fatty liver disease with or without diabetes: An updated systematic review of randomized controlled trials [J]. Diabetes Metab. 2020; 46 (6): 427-441.

[14] Angulo P, Kleiner D. E, Dam-Larsen S. Liver fibrosis, but no other histologic features, is associated with long-term outcomes of patients with nonalcoholic fatty liver disease [J]. Gastroenterology. 2015; 149 (e310): 389-397.

[15] Barbero-Becerra VJ, Santiago-Hernandez JJ, Villegas-Lopez FA, et al. Mechanisms involved in the protective effects of metformin against nonalcoholic fatty liver disease [J]. Current medicinal chemistry 2012, 19 (18): 2918-2923.

[16] Viollet B, Guigas B, Sanz Garcia N, et al. Cellular and molecular mechanisms of metformin: an overview [J]. Clinical science (London, England: 1979) 2012, 122 (6): 253-270.

[17] Huypens P, Quartier E, Pipeleers D, et al. Metformin reduces adiponectin protein expression and release in 3T3-L1 adipocytes involving activation of AMP activated protein kinase [J]. European journal of pharmacology 2005, 518 (2-3): 90-95.

[18] Kita Y, Takamura T, Misu H, et al. Metformin prevents and reverses inflammation in a non-diabetic mouse model of nonalcoholic steatohepatitis [J]. PloS one 2012, 7 (9): e43056.
[19] Garinis GA, Fruci B, Mazza A, et al. Metformin versus dietary treatment in nonalcoholic hepatic steatosis: a randomized study [J]. International journal of obesity (2005) 2010, 34 (8): $1255-1264$.

[20] Haukeland JW, Konopski Z, Eggesbø HB, et al. Metformin in patients with non-alcoholic fatty liver disease: a randomized, controlled trial $[\mathrm{J}]$. Scandinavian journal of gastroenterology 2009, 44 (7): 853-860.

[21] Loomba R, Lutchman G, Kleiner DE, et al. Clinical trial: pilot study of metformin for the treatment of non-alcoholic steatohepatitis [J]. Alimentary pharmacology \& therapeutics 2009, 29 (2): 172-182.

[22] Shields WW, Thompson KE, Grice GA, et al. The Effect of Metformin and Standard Therapy versus Standard Therapy alone in Nondiabetic Patients with Insulin Resistance and Nonalcoholic Steatohepatitis (NASH): A Pilot Trial [J]. Therapeutic advances in gastroenterology 2009, 2 (3): 157-163.

[23] Tiikkainen M, Häkkinen AM, Korsheninnikova E, et al. Effects of rosiglitazone and metformin on liver fat content, hepatic insulin resistance, insulin clearance, and gene expression in adipose tissue in patients with type 2 diabetes [J]. Diabetes 2004, 53 (8): 2169-2176.

[24] Nair S, Diehl AM, Wiseman M, et al. Metformin in the treatment of non-alcoholic steatohepatitis: a pilot open label trial [J]. Alimentary pharmacology \& therapeutics 2004, 20 (1): 23-28.

[25] Bugianesi E, Gentilcore E, Manini R, et al. A randomized controlled trial of metformin versus vitamin $\mathrm{E}$ or prescriptive diet in nonalcoholic fatty liver disease [J]. The American journal of gastroenterology 2005, 100 (5): 1082-1090.

[26] Chen HP, Shieh JJ, Chang CC, et al. Metformin decreases hepatocellular carcinoma risk in a dose-dependent manner: population-based and in vitro studies [J]. Gut 2013, 62 (4): 606-615.

[27] Lin CH, Kohli R. Emerging New Diagnostic Modalities and Therapies of Nonalcoholic Fatty Liver Disease [J]. Curr Gastroenterol Rep. 2020; 22 (10): 52.

[28] Geng Y, Hernandez Villanueva A, Oun A, Buist-Homan M, Blokzijl H, Faber KN, et al. Protective effect of metformin against palmitate-induced hepatic cell death [J]. Biochim Biophys Acta Mol Basis Dis. 2020; 1866 (3): 165621.

[29] Lavine JE, Schwimmer JB, Van Natta ML, et al. Effect of vitamin $\mathrm{E}$ or metformin for treatment of nonalcoholic fatty liver disease in children and adolescents: the TONIC randomized controlled trial [J]. Jama 2011, 305 (16): 1659-1668.

[30] Omer Z, Cetinkalp S, Akyildiz M, et al. Efficacy of insulin-sensitizing agents in nonalcoholic fatty liver disease $[\mathrm{J}]$. European journal of gastroenterology \& hepatology 2010, 22 (1): $18-23$. 